\title{
Quantitative reasoning skills in math methods
}

\author{
Michael Loverude ${ }^{1}$ \\ ${ }^{1}$ California State University Fullerton, Department of Physics, \\ MH-611, Fullerton, CA 92834
}

\begin{abstract}
Many upper-division physics courses have as goals that students should 'think like a physicist.' While this is often not defined, most would agree that thinking like a physicist includes quantitative reasoning skills: considering limiting cases, dimensional analysis, and using approximations. However, there is often relatively little curricular support for these practices and many instructors do not assess them explicitly. As part of a project to investigate student learning in math methods, we have developed a number of written questions testing these skills. Although there are limitations to assessing these skills with written questions, they can provide insight to the extent to which students can apply a given skill when prompted, even if they do not help understand how and when students choose to activate these skills.
\end{abstract}

PACS: 01.40.Fk, 01.40.G-, 02.30.-f

\section{INTRODUCTION}

This work is part of a collaboration to investigate student learning and application of mathematics in the context of upper-division physics courses, particularly the math methods courses offered in many physics departments. Our project seeks to study student conceptual understanding in upper-division physics courses, investigate models of transfer, and to develop instructional interventions to assist student learning.

While PER has primarily focused on introductory-level courses, there are increasing efforts to expand into the upper division [1]. The core sophomore- and junior-level courses taken by most physics majors have begun to receive the attention of researchers and curriculum developers. One key course that remains under-researched (with a few exceptions [2]) is the intermediate mathematical methods course that is taught by most departments. This course, which we will describe simply as Math Methods, is typically intended to bridge introductory-level physics and calculus content and core upper-level courses in electricity and magnetism and classical and quantum mechanics.

Typically the learning goals for courses of this nature focus on content goals, but often the courses also have stated or implicit goals that go beyond specific physics and mathematics context. For example, students in these courses are expected to 'think like a physicist' when solving quantitative problems. However, despite its seeming importance, this phrase is not always operationally defined. Examples of skills that might be included in this term include connecting physical intuition with mathematics, checking units and performing dimensional analysis, considering limiting cases, and using approximations. While instructors value these skills, and there has been some previous discussion of them [3], their value is often left implicit and they are not often explicitly taught or assessed.
In this portion of the project, we have sought to develop written tasks that ask students to apply the skills mentioned above and to document student responses to these tasks. For the purpose of this paper, we will refer to questions designed to assess several quantitative reasoning skills. The set of these skills is not intended to be complete, but we have identified several that appear to be relevant as starting points; examples of tasks related to several of these are shown below:

- Using dimensional / unit analysis

- Testing expressions with limiting cases

- Using approximations, e.g., with Taylor series

- Identifying errors in solutions

- Predicting the effects of problem changes

An example of the last task has been described previously [4]. A key goal of our project is to develop a series of tasks suitable for use in the math methods course that focus on the development of quantitative reasoning skills across multiple math and physics domains.

\section{BACKGROUND AND CONTEXT}

\section{A. Theoretical background}

This portion of our project is driven by practice, and we are seeking to learn what is difficult for students and develop instructional interventions. The theoretical framework guiding our analysis for this portion is "identifying student difficulties" [5], though other parts of the project reflect a resources approach and a transfer in pieces framework [6].

A growing body of work in PER has examined student use of mathematics in physics. In particular, several models have been proposed to describe student use of mathematics. In each of these models, successfully executing the mathematical procedure in question is only one element of success. For example, Wilcox et al have proposed the ACER framework 'to guide and structure investigations of 
students' difficulties with the sophisticated mathematical tools used in their physics classes.' [7] In this framework, students must activate the appropriate mathematical tool, construct a model, execute the math, and then reflect on results. The current study focuses only on the execution phase. For example, in some of the problems below, students are prompted to determine the units of a quantity; we cannot thus know how many students would spontaneously activate this skill to solve the problem. Qualitative studies of students in the process of solving problems can help to give insights into when and how students activate such resources and at what phase of problem solving they are employed. This project has a more modest goal, asking whether students can successfully apply skills when their use is explicitly cued.

\section{B. Context for research}

This work has taken place in the context Mathematical Methods of Physics, a course taught at California State University Fullerton, a large public comprehensive university serving a diverse student population. The course is required for physics majors and is a prerequisite for upper-division theory courses; for most students it is one of the first upper-division courses taken. The course uses the text by Boas [8] and covers a fairly standard list of topics. It meets for two 75-minute blocks per week. The course has as prerequisites three semesters of calculus, and most students have completed at least two semesters of introductory physics. The author has taught the course six times, with enrollments between 12 and 19. The Evaluate the Expressions task from part IIIA was posed on ungraded quizzes before instruction $(\mathrm{N}=47)$; multiple sections had the same problem. In contrast, the Determine the Units tasks are a variety of problems posed on course examinations after instruction; total sample is $\sim 50$ but individual tasks have smaller sample sizes.

\section{RESEARCH TASKS AND STUDENT RESPONSES}

For the purpose of this paper, we will focus on two types of task that are illustrative of the quantitative reasoning skills that we are describing. The first task will involve the evaluation of mathematical expressions for correctness, and will be based primarily on an ungraded quiz posed on the first day of instruction in the math methods course. The second task involves identifying units or dimensions for parameters in mathematical expressions or equations. Examples relating to this skill will be drawn from student responses to questions on course examinations throughout the semester.
Student written responses were examined and coded. Answers were coded for the correctness of the answer as well as the details of the student approach and explanation. Responses are tentatively assigned to categories that arise iteratively according to the major themes identified in the data. For the explanations, we use a grounded theory approach in which the entire data corpus is examined for common trends, and all data are reexamined to group them into the defined categories.

\section{A. Evaluate the expressions task}

The first of the tasks that we have used involves evaluating three mathematical expressions for correctness. The problem is posed on the first day of Math Methods on an ungraded quiz; the tasks are subsequently explored in a large group discussion while the tasks projected on a screen. The task describes an Atwood's machine with a massive pulley (see Figure 1). Students are shown three potential expressions for the acceleration of one of the two blocks and asked for each to determine whether the expression could be correct. (All are incorrect.) The problem has been administered in three sections of math methods $(N=47)$ on the first day of instruction.

The problem is different from many tasks that students have encountered to this point. It does not ask students to solve the problem, and has no numerical values. The expectation is that students will use quantitative reasoning to arrive at an answer; e.g., they might check whether there are reasonable circumstances in which the expression becomes unphysical. For example, in the second expression, if $\mathrm{M} / 2=\mathrm{m}_{1}+\mathrm{m}_{2}$ the denominator would vanish and acceleration would be infinite. In all cases, students can relate the expression to the motion that would result from this physical situation. For example, a student may reason that if the masses were equal, the system would not accelerate, and that the greater the difference in the two masses, the greater the acceleration.

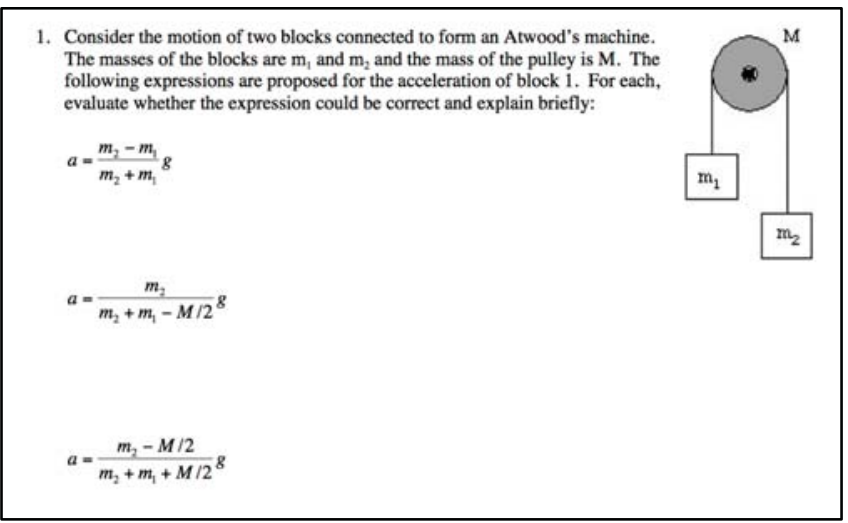

FIG 1. Evaluate the expressions task. 
TABLE 1. Common codes for evaluate the expression task. The codes were not exclusive; e.g., a student response might include both mechanism and mass difference $(N=47)$.

\begin{tabular}{c|l|c}
\hline Code & Description & Responses \\
\hline Solution & Attempted to solve problem & $10-15 \%$ \\
\hline Variables & $\begin{array}{l}\text { Presence or absence of } \\
\text { variables in expression }\end{array}$ & $20-50 \%$ \\
\hline Mechanism & $\begin{array}{l}\text { Described physical } \\
\text { mechanism (forces, energy) }\end{array}$ & $\sim 15 \%$ \\
\hline Limiting & Described limiting case(s) & $<5 \%$ \\
\hline
\end{tabular}

\section{Data analysis}

Student responses were coded iteratively based on both the correctness of their assessment and the explanation used in support. In the final version of the rubric, twelve distinct but not exclusive codes were used, with an 'other/blank' category used when students provided no intelligible explanation. Of the twelve codes, only a few were commonly used; the three most common codes as well as the 'expected' (limiting) answer are shown in Table I.

While some of the codes that emerged were expected, many were not. For example, a few students evaluated correctness based on whether the expression would have the correct sign, although no coordinate axis is specified. Written explanations may not indicate fully the underlying reasoning that students are using. For example, a few students stated that the rotational inertia of the massive pulley would decrease the acceleration and others simply noted the presence or absence of the pulley mass in the expression. We cannot be sure whether the students in two groups were using similar reasoning.

\section{Summary of student responses}

This task is challenging for students. Only one student offered a completely correct solution. Ten others identified all three solutions as incorrect but with incomplete or incorrect explanations. About $10 \%$ of students were coded as 'blank' for the first expression, and $20 \%$ for the second. Some explanations indicated lack of confidence:

...the mass of the pulley needs to be incorporated (although to tell the truth I am not sure how).

The approaches used by students varied considerably, and while many did give explanations that call upon physical intuition or an attempt to parse the mathematical expression, others seemed to respond as though this task were a more typical end of chapter problem. About $10 \%$ of the students solved the problem directly, and a few others performed algebraic manipulations of the given expressions. A few responses appeared as though they were to a multiple-choice question, with a circle or check mark next to one expression. One student wrote that the first response 'Needs the pulley!' and circled the second response, writing 'This one!!!' A few students mentioned remembered, or partially remembered, results:
"This one looks incorrect because it is adding $m / 2$ to the bottom and subtracting from the top; my very rusty memory only recalls subtracting from the bottom."

While remembering information can be valuable, the intent of these tasks is not to recall equations. Students may need to have the purpose of this activity framed very explicitly.

In addition to the difficulty of evaluating the correctness of the expressions, only a handful of students explained using quantitative reasoning as described above. As an example of a response that we coded in this category, one student wrote, 'No, if $\mathrm{m}_{2}=0 \mathrm{~kg}$ then this formula makes $\mathrm{a}=$ $0 \mathrm{~m} / \mathrm{s}^{2}$ which is clearly not true.'

A very common code reflected responses in which explanations referred to the presence or absence of variables: "This could be correct because all relevant variables are used." For the first expression, most students gave explanations that referred to the absence of the mass of the pulley. A few ( $\sim 5 \%)$ stated that the expression was incorrect because of the absence of $\mathrm{M}$, but the more common response was to state that the expression would be correct if the mass of the pulley is negligible.

Several student responses described a physical mechanism for the motion. A smaller group reflected an attempt to reconcile the mathematical form of the expression with this sense of physical mechanism. The most obvious examples of this were students who referred explicitly to the presence or absence of a term with the difference in masses. These responses included some in which the presence of this term was noted:

Correct [first expression]; $m_{2}$ is countering $m_{1}$ so $m_{1}$ is accelerating at a portion of $g$

as well as some rejecting expressions due to its absence:

...I don't think [the second expression] is correct

because there is no comparison of $m_{1}$ and $m_{2}$ in the numerator $\left(m_{2}-m_{1}\right)$

A few students gave explanations that reflected similar reasoning but with respect to other quantities:

this [second] expression raises the value of acceleration as the mass of the pulley increases leading me to believe this is incorrect.

\section{B. Determine the units tasks}

In this section we include a series of questions posed on course examinations. We added to standard questions a part in which students were asked to state the dimensions or units of a variable in a problem (see Figure 2). As students offered little in the way of explanations, data were coded primarily on student answers, though we examined student calculations accompanying correct and incorrect answers.

In many of the problems, identifying the appropriate units was straightforward. For example a task was embedded in a polar coordinates problem posed to three sections of the course $(N=41)$. In each case students were asked to identify the appropriate units for three quantities, including one length and one angular velocity (the third 
The temperature distribution will satisfy the heat flow equation $\frac{\partial^{2}}{\partial x^{2}} \tau-\frac{1}{\alpha^{2}} \frac{\partial}{\partial t} \tau=0 . \quad$ Assume that the temperature in the region is a product of two terms, $\tau(x, t)=X(x) T(t)$ so that

$\frac{1}{X} \frac{\partial^{2}}{\partial x^{2}} X=\frac{1}{T} \frac{1}{\alpha^{2}} \frac{\partial}{\partial t} T=\kappa$.

- What are the dimensions (units) of the constant $\kappa$ ?

of the constant $\alpha$ ?

FIG 2. A sample Determine the Units task, part of a longer problem on partial differential equations.

quantity differed among the three versions). The length was answered correctly by $90 \%$ of students. For the angular frequency, $75 \%$ of answers were coded as correct (1/s, radians/s, 1/time, Hz, radians/time). Answers of ' $\mathrm{rad} /$ s' were coded separately from answers of ' $1 / \mathrm{s}$ ' as it was not clear whether students recognized that radians are dimensionless. In one version of the problem, $(N=17)$ students were asked about the units of the quantity $b$ in the expression $r=R_{0^{-}} b \theta ; 7$ students answered with units of meters (or distance), and 7 others answered meters / radian (or distance / radian).

Other problems involving unit identification were more difficult for students; expressions involving quantities other than distance and time seem to be more challenging for students. For example, only 4 of 15 students in one section correctly identified the units of two quantities in an expression for a potential energy. Problems with derivatives and integrals have proven to be difficult for students. When asked to find the units of the constant $\mathrm{k}$ in the differential equation $\mathrm{d} V / \mathrm{d} t=-k A$ (with $V$ volume and $A$ area), $58 \%$ of the students (one section, $N=17$ ) answered correctly. In the task in Figure 2 only around $15 \%$ of students (three sections, $N=38$ ) have correctly identified the units of the constants $\kappa$ and $\alpha$. Student work suggests that, for example, the units resulting from the second spatial derivative of $\mathrm{X}(\mathrm{x})$ are not clear to many students. A few students appear to be confused by notation; for example, assigning to $V$ units of velocity rather than volume.

[1] M. Loverude and B. Ambrose, "Editorial: Focused Collection: PER in Upper-Division Physics Courses, Phys Rev ST-PER (in press).

[2] SEI: Science Education Initiative at U. Colorado, http://www.colorado.edu/sei/departments/physics.htm

[3] E. F. Redish, Proceedings of World View on Physics Education (2005).

[4] M.E. Loverude, AIP Conference Proceedings 1413 (2011).

\section{DISCUSSION}

Examining student responses to both types of problems lead to a few tentative conclusions. This portion of the project is in initial stages, and further research is needed.

First, many students entering the math methods course do not successfully reason quantitatively even with tasks designed to elicit this reasoning. The response given by some students suggest that they do not recognize that the tasks shown require them to step away from solving the problem directly or remembering its answer in order to reason whether a solution might be correct. Relatively few students spontaneously examined the expressions for special cases of the variables in the problem or related to a sense of physical mechanism.

Second, even after instruction many students struggle to identify the units in some of the expressions commonly encountered in the class. While units are a fundamental part of a physicist's toolkit, many students have difficulty with units as expressions get further from familiar quantities, as notation becomes more complicated and as mathematical operations like differentiation are included.

Finally, given that physicists value the quantitative skills described, there is a need for tasks that can be used in instruction and assessment. Redish and Kuo [9] have recently written that students "need to learn a component of physics expertise not present in math class-tying those formal mathematical tools to physical meaning...We as physics instructors must explicitly foster these components of expert physics practice to help students succeed in using math in physics." Yet the majority of problems in the course text are merely mathematical exercises that do not explicitly address these reasoning skills. Our data suggest that students need help in developing these skills.

\section{ACKNOWLEDGEMENTS}

This work is part of a collaboration with John Thompson (University of Maine), Joe Wagner (Xavier University) and Warren Christensen (North Dakota State University). The author acknowledges student researcher Marlene Vega (CSUF/NDSU REU). This work is supported by the NSF through grant PHYS-1405616; opinions, findings, and conclusions are those of the author and do not necessarily reflect the views of the NSF.

[5] P. Heron, "Enrico Fermi" Course CLVI, (IOS Press, Amsterdam, 2004), pp. 341-350

[6] D. Hammer, Am. J. Phys. 68, S52 (2000), Wagner Cogn. and Instr. 24, 1-71 (2006).

[7] B Wilcox et al, Phys. Rev. ST-PER 9, 0201191 (2013).

[8] M. Boas, Mathematical Methods in the Physical Sciences, Wiley (2006).

[9] E. Redish \& E. Kuo, Sci and Educ 24, 561-590 (2015). 